Recepción: 23 / 10 / 2017

Aceptación: 26 / $11 / 2017$

Publicación: 15/ 12/ 2017

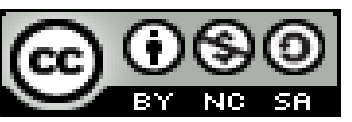

Ciencias económicas y empresariales

Artículo de investigación

\title{
Modelo de gestión de mantenimiento para las subestaciones y líneas de subtransmisión de CNEL EP unidad de negocio Esmeraldas
}

\section{Maintenance management model for substations and subtransmission lines of CNEL EP business unit Esmeraldas}

\section{Modelo de gerenciamento de manutenção para subestações e linhas de subtransmissão da unidade de negócio CNEL EP Esmeraldas}

Roddy A. Quiñónez-Ramoz ${ }^{\mathrm{V}}$

roddyalfredo@yahoo.com

Nicomedes L. Ordoñez-Zúñiga VI

nicoleonidas@yahoo.com

Neiva M. Quiñónez-Becerra VII

neivimary@hotmail.com
Simón R. Sánchez-Orozco ${ }^{\mathrm{I}}$ saymonsanz@hotmail.com

Luis E. Hidalgo-Solórzano II lehidalgo@hotmail.com

Jorge D. Mercado-Bautista ${ }^{\text {III }}$ mercadobautista@gmail.com

Violeta E. Reyes-Bone violetaelizabethreyesbone@yahoo.com

Correspondencia: saymonsanz@hotmail.com

I. Ingeniero Eléctrico, Docente Universidad Técnica Luis Vargas Torres de Esmeraldas, Esmeraldas, Ecuador.

II. Magister en Seguridad y Prevención de Riesgos del Trabajo, Ingeniero de Mantenimiento, Docente Universidad Técnica Luis Vargas Torres de Esmeraldas, Esmeraldas, Ecuador.

III. Ingeniero Mecánico, Docente Universidad Técnica Luis Vargas Torres de Esmeraldas, Esmeraldas, Ecuador.

IV. Ingeniero Mecánico, Docente Universidad Técnica Luis Vargas Torres de Esmeraldas, Esmeraldas, Ecuador.

v. Magister en Gestión Ambiental, Ingeniero Químico, Docente Universidad Técnica Luis Vargas Torres de Esmeraldas, Esmeraldas, Ecuador.

VI. Master of Science en Ingeniería, Ingeniero Mecánico en la Especialidad de Producción y Explotación de Máquinas, Docente Universidad Técnica Luis Vargas Torres de Esmeraldas, Esmeraldas, Ecuador.

VII. Ingeniera Químico, Docente Universidad Técnica Luis Vargas Torres de Esmeraldas, Esmeraldas, Ecuador. 


\title{
Resumen
}

Este proyecto técnico tiene como finalidad la elaboración de un Modelo de Programación de mantenimiento preventivo y predictivo, el cual sirve a todo el sistema de subtransmisión de CNEL EP Unidad de Negocio Esmeraldas comprendido por las líneas de subtransmisión y Subestaciones. Se realiza un recorrido por las subestaciones y líneas de subtransmisión que comprenden el sistema eléctrico de alta tensión de CNEL EP Unidad de negocio Esmeraldas, con el fin de ver las condiciones en que se encuentran, además se realizó un levantamiento de datos y se contó con información sobre los planes de mantenimiento de CNEL EP U N Esmeraldas. Se diseñan las tareas o actividades idóneas a realizar en cada uno de los equipos y elementos del sistema de subtransmisión, a partir de las normas establecidas. Se elabora una programación de mantenimiento predictivo y preventivo para las líneas de subtransmisión y subestaciones. Esta programación será de mucha utilidad en los futuros mantenimientos del sistema de subtransmisión de CNEL EP Unidad de Negocio Esmeraldas.

Palabras clave: modelo de programación de mantenimiento preventivo y predictivo; sistema de subtransmisión; CNEL EP Unidad de negocio Esmeraldas.

\begin{abstract}
The purpose of this technical project is to prepare a Predictive and Predictive Maintenance Programming Model, which serves the entire sub-transmission system of the CNEL EP Esmeraldas Business Unit comprised of the Subtransmission and Substation lines. A tour of substations and subtransmission lines comprising the high voltage electrical system of CNEL EP Business Unit Esmeraldas, in order to see the conditions in which they are located, in addition to a data survey was performed and was attended information on the maintenance plans of CNEL EP UN Esmeraldas. The tasks or suitable activities to be carried out in each of the equipment and elements of the subtransmission system are designed, based on the established standards. A predictive and preventive maintenance schedule is prepared for the subtransmission lines and substations. This programming will be very useful in the future maintenance of the subtransmission system of CNEL EP Esmeraldas Business Unit.
\end{abstract}

Keywords: preventive and predictive maintenance programming model; Subtransmission system; CNEL EP Business unit Esmeraldas. 


\section{Resumo}

O objetivo deste projeto técnico é preparar um Modelo de Programação de Manutenção Preditiva e Preditiva, que serve todo o sistema de sub-transmissão da Unidade de Negócios da CNEL EP Esmeraldas, composta pelas linhas Subtransmissão e Subestação. Um passeio de subestações e linhas de subtransmissão que compõem o sistema elétrico de alta tensão da Unidade de Negócios da CNEL EP Esmeraldas, para ver as condições em que estão localizadas, além de um levantamento de dados foi realizado e foi fornecido com Informações sobre os planos de manutenção do CNEL EP UN Esmeraldas. As tarefas ou atividades adequadas a serem realizadas em cada um dos equipamentos e elementos do sistema de subtransmissão são projetadas, com base nos padrões estabelecidos. Um cronograma de manutenção preditiva e preventiva é preparado para as linhas e subestações de subtransmissão. Esta programação será muito útil na futura manutenção do sistema de subtransmissão da Unidade de Negócios CNEL EP Esmeraldas. Palavras chave: modelo de programação de manutenção preventiva e preditiva; Sistema de subtransmissões; CNEL EP Unidade de negócios Esmeraldas.

\section{Introducción}

El 13 de marzo del 2013, el Presidente de la República de Ecuador, Economista Rafael Correa Delgado emitió el Decreto Ejecutivo No. 1459, constituyendo la Empresa Eléctrica Pública Estratégica Corporación Nacional de Electricidad CNEL EP, la cual desde ese momento tuvo como misión prestar los servicios públicos de distribución y comercialización de energía eléctrica con el fin de contribuir al desarrollo del país y generar a los consumidores bienestar. Esto lo logra con innovación, un comprometido talento humano, tecnología y respetando al medio ambiente.

En la actualidad el país está culminando un proceso de cambio de la matriz energética y de esta forma lograr el Objetivo 11, Política 11.1 del Plan Nacional del Buen Vivir 2013-2017 el cual busca reestructurar la matriz energética bajo criterios de transformación de la matriz productiva, inclusión, calidad, soberanía energética y sustentabilidad, con incremento de la participación de energía renovable. (SENPLADES, 2013)

Al lograr dicho objetivo los beneficiados serán los consumidores finales de tipo residencial, comercial o industrial ya que contará con un servicio eléctrico bajo estándares de calidad y 
continuidad. Para tener este beneficio debe de existir un sistema eléctrico de potencia en óptimas condiciones. Las subestaciones y líneas de sub-transmisión se consideran la columna vertebral para culminar con el servicio eléctrico deseado ya que por ellas pasa la energía generada antes de ser distribuida a los diferentes tipos de abonados.

Una subestación es uno de los elementos que conforman un sistema eléctrico de potencia el cual tiene como función modificar los valores de corriente y tensión, además permite la transmisión y distribución de energía eléctrica sirviendo como punto de interconexión. (Pérez, 2011)

El mantenimiento es el conjunto necesario de tareas para que un determinado equipo sea restaurado o conservado de tal manera que permanezca con una condición específica acordada. (www.solomantenimiento,com, s.f)

Mantenimiento es una determinada acción para garantizar mejoras como su productividad, funcionalidad, confort, imagen, seguridad, higiene y salubridad en los aspectos operativos de relevancia de un sistema. (San clemente \& Nieto Alvarado, 2010)

Sin duda el principal el objetivo de las empresas dedicadas a brindar un servicio de energía eléctrica es de garantizar la continuidad del servicio de manera confiable y de calidad. Los sistemas eléctricos son propensos a realizar sus funciones en condiciones desfavorables y por ende pueden sufrir fallas en un determinado momento de naturaleza mecánica, eléctrica u otra.

Para afrontar estas condiciones las empresas invierten grandes cantidades económicas en equipos de control, medición, monitoreo, entre otros. Por lo mencionado anteriormente si llegase a ocurrir algún tipo de falla, representaría para las empresas también grandes pérdidas económicas.

En Ecuador, el proceso de cambio de la Matriz Energética Nacional se identifica con el deseo de cumplir con el objetivo de brindar un servicio continuo, confiable y de calidad, por lo cual todas las empresas generadoras, transmisoras y distribuidoras de energía eléctrica deberán contar necesariamente con modelos de gestión de mantenimiento para dichos equipos y elementos del sistema que son propensos a fallas. (Francisco \& Ochoa, 2008) 
En los treinta últimos años el tema mantenimiento ha evolucionado más que cualquier otra disciplina gerencial, se lo ha dejado de ver como un gasto para ahora apreciarlo como una inversión. El aumento de la población conlleva a tener a las empresas de generación, transmisión y distribución una mayor demanda de su producto para utilizarlo en el ámbito residencial, comercial e industrial. Por tanto, se construyen constantemente empresas de las antes mencionadas y he aquí la necesidad de realizar un correcto mantenimiento a sus instalaciones para garantizar la calidad del servicio. (Martínez, 2010)

Es evidente la importancia de un modelo de gestión de mantenimiento en las subestaciones y líneas de subtransmisión de CNEL EP., con esto se evitaría determinadas fallas para minimizar tiempos muertos en lo referente a producción y así satisfacer las necesidades de sus clientes.

Este trabajo tiene como objetivo general: Presentar un modelo de gestión de mantenimiento para las subestaciones y líneas de subtransmisión de CNEL EP Unidad de Negocio Esmeraldas.

\section{Metodología}

En el proceso del proyecto se utilizan los datos brindados por el departamento de subestaciones. Los mismos se analizan y sintetizan y se realizan inducciones y deducciones.

\section{Desarrollo}

Lo primero que se hizo fue determinar el estado actual de los planes de mantenimiento e infraestructura de las subestaciones y líneas de subtransmisión de CNEL EP Unidad de negocio Esmeraldas, lo que evidenció problemas en este sentido.

En segundo lugar, se establece el banco de tareas para cada tipo de mantenimiento a realizar. Para poder brindar un servicio continuo de energía eléctrica hay que tener un plan de mantenimiento muy bien estructurado ya que el objetivo es, la operación ininterrumpida del sistema de subtransmisión, por esta razón se puede deducir lo protagónico de un mantenimiento adecuado. 
A continuación, se detallan los bancos de tareas y normas en las cuales se amparan los distintos tipos de mantenimientos. Se utilizan las abreviaciones: $\mathrm{A}=$ Anual, Semestral $=\mathrm{SM}$, Mensual $=$ $\mathrm{M}, \mathrm{CR}=$ Cada que se requiera.

Tabla 1 Banco de tareas para mantenimiento predictivo en transformadores de potencia.

\begin{tabular}{|l|l|l|}
\hline \multicolumn{2}{|l|}{ Banco de tareas para Transformadores de Potencia. } & Frecuencia \\
\hline $\mathbf{1}$ & Factor de potencia al aislamiento de devanados. & $\boldsymbol{A}$ \\
\hline $\mathbf{2}$ & Relación de transformación. & $\boldsymbol{A}$ \\
\hline $\mathbf{3}$ & Resistencia de aislamiento en devanados. & $\boldsymbol{A}$ \\
\hline $\mathbf{4}$ & Termografia. & $\boldsymbol{A}$ \\
\hline $\mathbf{5}$ & Collar caliente a boquillas de alta tensión. & $\boldsymbol{A}$ \\
\hline $\mathbf{6}$ & Prueba de aislamiento de los devanados. & $\boldsymbol{A}$ \\
\hline $\mathbf{7}$ & Prueba Rigidez dieléctrica Aceite Transformadores. & $\boldsymbol{A}$ \\
\hline $\mathbf{8}$ & Medición y análisis de carga del transformador. & $\boldsymbol{A}$ \\
\hline $\mathbf{9}$ & Medición de la resistencia óhmica de los devanados. & $\boldsymbol{A}$ \\
\hline $\mathbf{1 0}$ & Medidas del nivel de ruido. & $\boldsymbol{A}$ \\
\hline $\mathbf{1 1}$ & $\begin{array}{l}\text { Pruebas de Factor de potencia, rigidez dieléctrica, Resistividad, Coloración y acidez al } \\
\text { aceite aislante. }\end{array}$ & $\boldsymbol{A}$ \\
\hline
\end{tabular}

Fuente: http://dispac.com.co/wp-content/uploads/2015/05/ANEXO-18-A-MANUAL-DEMANTENIMIENTO-PARA-SUBESTACIONES.pdf

Tabla 2 Banco de tareas para mantenimiento predictivo en interruptores de potencia.

\begin{tabular}{|l|l|l|}
\hline \multicolumn{2}{|l|}{ Banco de tareas para Interruptores de Potencia. } & Frecuencia \\
\hline $\mathbf{1}$ & Tiempo de Operación & A \\
\hline $\mathbf{2}$ & Medida de Desplazamiento & $\mathbf{2 A}$ \\
\hline $\mathbf{3}$ & Resistencia de Aislamiento & $\mathbf{2} \mathrm{A}$ \\
\hline $\mathbf{4}$ & Consumo y Resistencia de Bobinas de Cierre y Apertura & $\mathbf{2} \mathrm{A}$ \\
\hline $\mathbf{5}$ & Medida de Sobre alcance y Rebote & $\mathbf{2} \mathrm{A}$ \\
\hline $\mathbf{6}$ & Factor de potencia & $\mathbf{2} \mathrm{A}$ \\
\hline $\mathbf{7}$ & Consumo y Resistencia del motor & $\mathbf{2 A}$ \\
\hline $\mathbf{8}$ & Sincronismo de apertura y cierre. & $\mathbf{2 A}$ \\
\hline
\end{tabular}




\begin{tabular}{|c|c|c|}
\hline 9 & Prueba de simultaneidad de polos al cierre a la apertura. & $2 A$ \\
\hline 10 & Termografía. & $2 A$ \\
\hline 11 & $\begin{array}{l}\text { Medida de velocidad y desplazamiento, de indicación del estado del accionamiento } \\
\text { mecánico. }\end{array}$ & $2 A$ \\
\hline 12 & Medida de resistencia de contacto dinámica. & $2 A$ \\
\hline 13 & $\begin{array}{l}\text { Medida del punto de rocio, está asociado al grado de pureza del SF6 en cuanto a su } \\
\text { contaminación. }\end{array}$ & $M$ \\
\hline 14 & Análisis químico de subproductos de la descomposición del gas SF6. & $M$ \\
\hline 15 & $\begin{array}{l}\text { Análisis de propiedades eléctricas y fisicas del gas, similar a los ensayos al aceite de } \\
\text { transformadores. Humedad y rigidez dieléctrica. }\end{array}$ & $2 A$ \\
\hline 16 & Collar caliente a boquillas & $2 A$ \\
\hline
\end{tabular}

Fuente: http://dispac.com.co/wp-content/uploads/2015/05/ANEXO-18-A-MANUAL-DE-

\section{MANTENIMIENTO-PARA-SUBESTACIONES.pdf}

Tabla 3 Banco de tareas para Mantenimiento Predictivo en Seccionadores de Potencia.

\begin{tabular}{|l|l|l|}
\hline \multicolumn{2}{|l|}{ Banco de tareas para Seccionadores de Potencia. } & Frecuencia. \\
\hline 1 & Pruebas de Aislamiento. & A \\
\hline 2 & Prueba de resistencia de contactos. & $A$ \\
\hline 3 & Verificación de cierre total y apertura total. & A \\
\hline
\end{tabular}

Fuente: http://dispac.com.co/wp-content/uploads/2015/05/ANEXO-18-A-MANUAL-DEMANTENIMIENTO-PARA-SUBESTACIONES.pdf

Tabla 4 Banco de tareas para Mantenimiento Predictivo de Transformadores de Corriente.

\begin{tabular}{|l|l|l|}
\hline \multicolumn{2}{|l|}{ Banco de tareas para Transformadores de Corriente. } & Frecuencia. \\
\hline $\mathbf{1}$ & Factor de Potencia. & $\mathbf{2}$ \\
\hline $\mathbf{2}$ & Verificación Resistencia de devanados & $\mathbf{A} \boldsymbol{A}$ \\
\hline $\mathbf{3}$ & Pruebas de Aislamiento. & $\mathbf{2} \boldsymbol{A}$ \\
\hline $\mathbf{4}$ & Verificación de la relación de transformación y polaridad. & $\mathbf{2} \boldsymbol{A}$ \\
\hline $\mathbf{5}$ & Curvas de saturación. & $\mathbf{2 A}$ \\
\hline $\mathbf{6}$ & Curva de Magnetización & $\mathbf{2 A}$ \\
\hline
\end{tabular}

Fuente: http://dispac.com.co/wp-content/uploads/2015/05/ANEXO-18-A-MANUAL-DEMANTENIMIENTO-PARA-SUBESTACIONES.pdf 
Tabla 5 Banco de tareas para Mantenimiento Predictivo en Transformadores de Potencial.

\begin{tabular}{|l|l|l|}
\hline \multicolumn{2}{|l|}{ Banco de tareas para Transformadores de Potencial o Tensión. } & Frecuencia. \\
\hline $\mathbf{1}$ & Pruebas de Aislamiento. & $\mathbf{2}$ \\
\hline $\mathbf{2}$ & Verificación de la relación de transformación y polaridad. & $\mathbf{A} \boldsymbol{A}$ \\
\hline $\mathbf{3}$ & Curvas de saturación. & $\mathbf{2 A}$ \\
\hline $\mathbf{4}$ & Curva de Magnetización. & $\mathbf{2 A}$ \\
\hline $\mathbf{5}$ & Factor de Potencia. & $\mathbf{A}$ \\
\hline $\mathbf{6}$ & Verificación Resistencia de devanados. & $\mathbf{2 A}$ \\
\hline 7 & Relación de Transformación & $\boldsymbol{A}$ \\
\hline
\end{tabular}

Fuente: http://dispac.com.co/wp-content/uploads/2015/05/ANEXO-18-A-MANUAL-DEMANTENIMIENTO-PARA-SUBESTACIONES.pdf

Tabla 6 Banco de tareas para Mantenimiento Predictivo a los Pararrayos.

\begin{tabular}{|l|l|l|}
\hline \multicolumn{2}{|l|}{ Banco de tareas para los Pararrayos. } & Frecuencia. \\
\hline 1 & Factor de Potencia. & $2 A$ \\
\hline 2 & Pruebas de aislamiento. & $2 A$ \\
\hline 3 & Prueba de corriente de fuga. & $2 A$ \\
\hline
\end{tabular}

Fuente: http://dispac.com.co/wp-content/uploads/2015/05/ANEXO-18-A-MANUAL-DEMANTENIMIENTO-PARA-SUBESTACIONES.pdf

Tabla 7 Banco de Tareas para Mantenimiento Predictivo en el Sistema de Puesta a Tierra.

\begin{tabular}{|l|l|l|}
\hline \multicolumn{2}{|l|}{ Banco de Tareas para el Sistema de Puesta a Tierra. } & Frecuencia \\
\hline 1 & Medida de tensiones de paso y contacto. & $2 \boldsymbol{A}$ \\
\hline 2 & Medida de la resistencia de puesta a tierra. & $2 \mathrm{~A}$ \\
\hline
\end{tabular}

Fuente: http://dispac.com.co/wp-content/uploads/2015/05/ANEXO-18-A-MANUAL-DEMANTENIMIENTO-PARA-SUBESTACIONES.pdf

Tabla 8 Banco de tareas para Mantenimiento Predictivo en Barrajes, Cables desnudos y conectores de alta.

\begin{tabular}{|l|l|l|}
\hline \multicolumn{2}{|l|}{ Banco de tareas para Barrajes, Cables desnudos y Conectores de alta. } & Frecuencia. \\
\hline 1 & Termografía. & 2 A \\
\hline
\end{tabular}


2

Pruebas de aislamiento.

$2 A$

Fuente: http://dispac.com.co/wp-content/uploads/2015/05/ANEXO-18-A-MANUAL-DE-

MANTENIMIENTO-PARA-SUBESTACIONES.pdf

Tabla 9 Banco de tareas para Mantenimiento Predictivo en Celdas de alto voltaje.

\begin{tabular}{|l|l|l|}
\hline \multicolumn{2}{|l|}{ Banco de tareas para Celdas de alto voltaje. } & Frecuencia. \\
\hline $\mathbf{1}$ & Pruebas de aislamiento al barraje. & A \\
\hline $\mathbf{2}$ & Pruebas a los interruptores. & $\boldsymbol{A}$ \\
\hline 3 & Termografia. & SM \\
\hline
\end{tabular}

Fuente: http://dispac.com.co/wp-content/uploads/2015/05/ANEXO-18-A-MANUAL-DE-

MANTENIMIENTO-PARA-SUBESTACIONES.pdf

Tabla 10 Banco de tareas para Mantenimiento Predictivo de Relés de Protecciones.

\begin{tabular}{|l|l|l|}
\hline \multicolumn{2}{|l|}{ Banco de tareas para Relés de Protecciones. } & Frecuencia. \\
\hline $\mathbf{1}$ & Comprobación de disparos y alarmas. & M \\
\hline $\mathbf{2}$ & Verificar ajuste de conexiones. & A \\
\hline $\mathbf{3}$ & Calibración de corrientes y de tiempos. & $\mathbf{2}$ A \\
\hline $\mathbf{4}$ & Pruebas dieléctricas. & A \\
\hline $\mathbf{5}$ & Termografia. & $\mathbf{2 A}$ \\
\hline
\end{tabular}

Fuente: http://dispac.com.co/wp-content/uploads/2015/05/ANEXO-18-A-MANUAL-DE-

MANTENIMIENTO-PARA-SUBESTACIONES.pdf

Tabla 11 Banco de tareas para Mantenimiento Predictivo en los Bancos de Baterías.

\begin{tabular}{|l|l|l|}
\hline \multicolumn{2}{|l|}{ Banco de tareas para los Bancos de Baterías. } & Frecuencia \\
\hline 1 & Pruebas de densidad. & $2 \mathrm{M}$ \\
\hline 2 & Pruebas de temperatura. & $\mathbf{2 M}$ \\
\hline
\end{tabular}

Fuente: http://dispac.com.co/wp-content/uploads/2015/05/ANEXO-18-A-MANUAL-DE-

MANTENIMIENTO-PARA-SUBESTACIONES.pdf

Tabla 12 Banco de tareas para Mantenimiento Preventivo a Transformadores de Potencia.

\section{Banco de tareas para Transformadores de Potencia.}

1 Verificar niveles de aceite en el conservador, bushings, y tanque (si lo hay).

\section{Frecuencia}

$\boldsymbol{M}$ 


\begin{tabular}{|c|c|c|}
\hline 2 & Verificar los bushings y terminales. & $M$ \\
\hline 3 & Verificar las lecturas de los medidores de temperatura. & $M$ \\
\hline 4 & Verificar existencia de fugas de aceites. & $M$ \\
\hline 5 & Verificar la operación de ventiladores y pruebas de funcionamiento. & $M$ \\
\hline 6 & Verificar la operación de calefactores. & $M$ \\
\hline 7 & Coger fallas de pintura. & $\boldsymbol{A}$ \\
\hline 8 & Verificar coloración del silicagel. & $M$ \\
\hline 9 & Verificar ruidos anormales. & $M$ \\
\hline 10 & Chequeo de conexión a tierra. & $S M$ \\
\hline 11 & Limpiar la porcelana de los bushings. & $A$ \\
\hline 12 & Verificar el ajuste de los terminales de los bushings y conexionado. & $\boldsymbol{A}$ \\
\hline 13 & Verificar el ajuste mecánico total del transformador. & $\boldsymbol{A}$ \\
\hline 14 & Realizar una prueba dieléctrica del aceite aislante. & SM \\
\hline 15 & Pruebas del factor de potencia del aceite. & $\boldsymbol{A}$ \\
\hline 16 & Pruebas del factor de potencia de devanados. & $\boldsymbol{A}$ \\
\hline 17 & Medición de resistencia de aislamiento devanados y pruebas de absorción dieléctrica. & $\boldsymbol{A}$ \\
\hline 18 & Medición de resistencia de aislamiento de los motores del sistema de enfriamiento. & $\boldsymbol{A}$ \\
\hline 19 & Pruebas de protección por elevación de temperatura. & $\boldsymbol{A}$ \\
\hline 20 & Pruebas de funcionamiento de la válvula de explosión. & $\boldsymbol{A}$ \\
\hline 21 & Pruebas químicas de acidez, tensión interfacial, contenido de humedad. & $C R$ \\
\hline 22 & Renovación o cambio del Silicagel. & $\boldsymbol{A}$ \\
\hline \multicolumn{3}{|c|}{ Mando a motor. } \\
\hline 23 & Revisar operación de calefactores. & $M$ \\
\hline 24 & Verificar ausencia de humedad en el gabinete. & $M$ \\
\hline 25 & Verificar el estado de dispositivos. & $M$ \\
\hline 26 & Chequear el nivel de aceite en el reductor principal. & $M$ \\
\hline 27 & Medición de resistencia de aislamiento del motor. & $2 A$ \\
\hline \multicolumn{3}{|c|}{ Regulador automático de voltaje (A.V.R.). } \\
\hline 28 & Verificar el estado del AVR, dispositivos, ausencia de humedad en el gabinete. & $M$ \\
\hline 29 & Verificación de voltajes de prueba. & $S M$ \\
\hline
\end{tabular}




\begin{tabular}{|c|c|c|}
\hline \multicolumn{3}{|c|}{ Cambiador de Taps bajo carga. } \\
\hline 30 & Verificar el nivel de aceite. & $M$ \\
\hline 31 & Controlar la estanqueidad de la tapa del regulador. & $M$ \\
\hline 32 & $\begin{array}{l}\text { Tomar lecturas del manómetro del filtro de aceite y verificar que no sea mayor que } \\
\text { cuatro bares. }\end{array}$ & $A$ \\
\hline 33 & Revisar operación de resistencia de calefacción. & $M$ \\
\hline 34 & Medición de resistencia de aislamiento del motor del filtro. & $2 A$ \\
\hline 35 & Observar fugas de aceite. & $M$ \\
\hline 36 & Inspección visual del mecanismo, posición del tap y pruebas de operación. & $S M$ \\
\hline 37 & Registrar la lectura del contador. & $M$ \\
\hline 38 & Realizar una prueba dieléctrica del aceite. & $A$ \\
\hline 39 & Verificar los lubricantes de los engranajes. & $A$ \\
\hline 40 & $\begin{array}{l}\text { Operar el LTC a pleno rango de Taps, observar el mecanismo, el indicador de taps y } \\
\text { fines de carrera. }\end{array}$ & $A$ \\
\hline 41 & Verificar el ajuste del conexionado. & $A$ \\
\hline 42 & Realizar una prueba de pérdidas dieléctricas en el aceite. & $A$ \\
\hline \multicolumn{3}{|c|}{ Relé Buchholz } \\
\hline 43 & Verificar que el relé este lleno de aceite. & $M$ \\
\hline 44 & Realizar pruebas de operación. & $A$ \\
\hline 45 & Verificar estanqueidad del relé. & $A$ \\
\hline \multicolumn{3}{|c|}{ Relé de Presión } \\
\hline 46 & Verificar el estado del relé, pruebas de operación y ausencia de humedad. & $A$ \\
\hline 47 & Verificar estanqueidad del relé. & $A$ \\
\hline
\end{tabular}

Fuente: Instituto Ecuatoriano de Electrificación (INECEL)

Tabla 13 Banco de tareas para Mantenimiento Preventivo a Interruptores de Potencia.

\section{Banco de tareas para Interruptores de Potencia.}

\begin{tabular}{|l|l|l|}
\hline $\mathbf{1}$ & Verificar niveles de aceite en el tanque y bushings. & $\boldsymbol{M}$ \\
\hline $\mathbf{2}$ & Inspección visual del gabinete de control y alrededores. & $\boldsymbol{M}$ \\
\hline $\mathbf{3}$ & Verificar la correcta operación de calefactores. & $\boldsymbol{M}$ \\
\hline $\mathbf{4}$ & Efectuar una prueba de operación (recomendado). & $\boldsymbol{A}$ \\
\hline
\end{tabular}

\section{Frecuencia}




\begin{tabular}{|c|c|c|}
\hline 5 & Efectuar una prueba dieléctrica del aceite aislante y factor de potencia. & $A$ \\
\hline 6 & Verificar la operación del contador y registros de lecturas. & $A$ \\
\hline 7 & Chequeo de bushings y limpieza. & $A$ \\
\hline 8 & $\begin{array}{l}\text { Realizar una prueba de carbón si la apariencia del aceite es dudosa, pruebas químicas } \\
\text { de acidez, tensión interfacial y humedad. }\end{array}$ & CR \\
\hline 9 & Revisar el motor de operación del comando del interruptor. & $A$ \\
\hline 10 & Verificar el ajuste mecánico de los componentes del gabinete de control. & $A$ \\
\hline 11 & Revisar el switch auxiliar y contactores. & $A$ \\
\hline 12 & Lubricar el mecanismo en los sitios necesarios. & $A$ \\
\hline 13 & Pruebas de tiempo de cierre y apertura. & $2 A$ \\
\hline 14 & Medición de resistencia de contactos y revisión de los mismos. & $A$ \\
\hline 15 & Realizar pruebas por protección. & $A$ \\
\hline 16 & Pruebas de factor de potencia totales. & $2 A$ \\
\hline 17 & Coger fallas de pintura. & $A$ \\
\hline 18 & Revisión de contactos y cámara de extinción de arco. & $5 \mathrm{~A}$ \\
\hline \multicolumn{3}{|c|}{ SF6 } \\
\hline 19 & Observar la presión del aire y posibles fugas, chequear la operación del compresor. & $M$ \\
\hline 20 & Drenar el agua en el reservorio de aire. & SEMANAL \\
\hline 21 & $\begin{array}{l}\text { Chequear la operación del compresor, observar ruidos anormales y la condición de las } \\
\text { correas y niveles de aceite. }\end{array}$ & $M$ \\
\hline 22 & Inspección visual y verificación de calefactores del gabinete de control. & $M$ \\
\hline 23 & Cambio de aceite del compresor (a las 200 horas de operación). & $5 \mathrm{~A}$ \\
\hline 24 & Chequear el contador de operaciones. & $A$ \\
\hline 25 & Inspección y engrase del mecanismo de trabajo. & $A$ \\
\hline 26 & Revisar contactores, switchs auxiliares, contactos auxiliares y conexionado. & $A$ \\
\hline 27 & Pruebas de resistencia de aislamiento de bushings. & $A$ \\
\hline 28 & Medición de resistencia de contactos. & $A$ \\
\hline 29 & Chequear bushings y limpiarlos. & $A$ \\
\hline 30 & $\begin{array}{l}\text { Realizar unas pruebas de operación local, remoto y por protección (eléctricas, aire y } \\
\text { gas). }\end{array}$ & $A$ \\
\hline 31 & Pruebas de factor de potencia. & $2 A$ \\
\hline
\end{tabular}




\begin{tabular}{|c|c|c|}
\hline 32 & Pruebas de tiempo de cierre y apertura. & $2 A$ \\
\hline 33 & Verificar hermeticidad de gas (SF6). & $M$ \\
\hline 34 & Verificar el consumo de aires en aperturas. & $\boldsymbol{A}$ \\
\hline 35 & Revisión de contactos y de la Unidad de ruptura. & CR \\
\hline 36 & Medición de la resistencia de aislamiento del motor del compresor. & $\boldsymbol{A}$ \\
\hline 37 & $\begin{array}{l}\text { Registro del tiempo de funcionamiento continúo del compresor (limite inferior al límite } \\
\text { superior). }\end{array}$ & $\boldsymbol{A}$ \\
\hline 38 & Pruebas de operación de la válvula de seguridad. & $A$ \\
\hline 39 & Verificar fugas de aceite del amortiguador. & $M$ \\
\hline
\end{tabular}

Fuente: Instituto Ecuatoriano de Electrificación (INECEL)

Tabla 14 Banco de tareas para Mantenimiento Preventivo a Seccionadores de Potencia.

\begin{tabular}{|c|c|c|}
\hline \multicolumn{2}{|r|}{ Banco de tareas Seccionadores de Potencia. } & \multirow{2}{*}{$\begin{array}{l}\text { Frecuencia } \\
M\end{array}$} \\
\hline 1 & Verificar visualmente la posición de las cuchillas y estado de aisladores. & \\
\hline 2 & Verificar el mecanismo del motor. & $M$ \\
\hline 3 & Verificar la operación de los calefactores. & $M$ \\
\hline 4 & Realizar una operación eléctrica y una manual. & $A$ \\
\hline 5 & $\begin{array}{l}\text { Operar manualmente y chequear el aislamiento de contactos, ranuras, topes, facilidad } \\
\text { de operación, presión de contactos, cable trenzado. }\end{array}$ & $\boldsymbol{A}$ \\
\hline 6 & Chequear los contactos para detectar desgaste de la capa de plata y chisporroteo. & $\boldsymbol{A}$ \\
\hline 7 & Limpiar los contactos y lubricarlos. & $\boldsymbol{A}$ \\
\hline 8 & Verificar el ajuste de tornillos, pernos, tuercas, pasadores, etc. & $\boldsymbol{A}$ \\
\hline 9 & Chequear los cuerpos de arcos o anillos equipotenciales y limpiar materiales desgaste. & $\boldsymbol{A}$ \\
\hline 10 & Inspeccionar y lubricar partes móviles. & $\boldsymbol{A}$ \\
\hline 11 & Verificar la puesta a tierra y observar algún daño. & $\boldsymbol{A}$ \\
\hline 12 & Limpieza de aisladores y coger fallas de pintura. & $\boldsymbol{A}$ \\
\hline \multicolumn{3}{|c|}{ Mecanismo del motor. } \\
\hline 13 & Revisión y limpieza de contactos auxiliares, circuitos del motor y fusibles. & $\boldsymbol{A}$ \\
\hline 14 & Chequear el conexionado y su ajuste. & $\boldsymbol{A}$ \\
\hline 15 & Inspeccionar y lubricar partes móviles. & $\boldsymbol{A}$ \\
\hline 16 & Inspeccionar el conmutador del motor y boquillas. & $A$ \\
\hline
\end{tabular}




\begin{tabular}{|c|l|l|}
\hline $\mathbf{1 7}$ & Chequear la operación correcta de seguros o interbloqueos. & $\boldsymbol{A}$ \\
\hline $\mathbf{1 8}$ & Medición de resistencia de aislamiento del motor. & $\boldsymbol{A}$ \\
\hline $\mathbf{1 9}$ & Medición de la resistencia de contactos. & $\boldsymbol{A}$ \\
\hline $\mathbf{2 0}$ & Medición, limpieza y lubricación del sistema de puesta a tierra. & $\boldsymbol{A}$ \\
\hline
\end{tabular}

Fuente: Instituto Ecuatoriano de Electrificación (INECEL)

Tabla 15 Banco de Tareas para Mantenimiento Preventivo en Transformadores de Potencial o Tensión

\begin{tabular}{|l|l|l|}
\hline \multicolumn{2}{|l|}{ Banco de Tareas para Transformadores de Potencial o Tensión. } & Frecuencia \\
\hline $\mathbf{1}$ & $\begin{array}{l}\text { Realizar una inspección visual de los bushings, tanques, niveles de aceite, estructuras y } \\
\text { puesta a tierra. }\end{array}$ & M \\
\hline $\mathbf{2}$ & Chequear la porcelana y observar despostillados, rajaduras, cementación y limpieza. & A \\
\hline $\mathbf{3}$ & Chequear tubería, accesorios, conexionado y fusibles. & A \\
\hline $\mathbf{4}$ & Pruebas del factor de potencia. & $\mathbf{2}$ \\
\hline $\mathbf{5}$ & Pruebas de resistencia de aislamiento. & $\boldsymbol{A}$ \\
\hline $\mathbf{6}$ & Coger fallas de pintura. & $\boldsymbol{A}$ \\
\hline
\end{tabular}

Fuente: Instituto Ecuatoriano de Electrificación (INECEL)

Tabla 16 Banco de Tareas para Mantenimiento Preventivo en Transformadores de Corriente.

\begin{tabular}{|l|l|l|}
\hline \multicolumn{2}{|l|}{ Banco de Tareas para Transformadores de Potencial o Tensión. } & Frecuencia \\
\hline $\mathbf{1}$ & $\begin{array}{l}\text { Realizar una inspección visual de los bushings, tanques, niveles de aceite, estructuras y } \\
\text { puesta a tierra. }\end{array}$ & M \\
\hline $\mathbf{2}$ & Chequear la porcelana y observar despostillados, rajaduras, cementación y limpieza. & A \\
\hline $\mathbf{3}$ & Chequear tubería, accesorios, conexionado y fusibles. & A \\
\hline $\mathbf{4}$ & Pruebas del factor de potencia. & $\mathbf{2}$ \\
\hline $\mathbf{5}$ & Pruebas de resistencia de aislamiento. & $\boldsymbol{A}$ \\
\hline $\mathbf{6}$ & Coger fallas de pintura. & $\boldsymbol{A}$ \\
\hline
\end{tabular}

Fuente: Instituto Ecuatoriano de Electrificación (INECEL)

Tabla 17 Banco de Tareas para Mantenimiento Preventivo a Pararrayos.

\begin{tabular}{|l|l|l|}
\hline \multicolumn{2}{|l|}{ Banco de tareas para los Pararrayos. } & Frecuencia \\
\hline 1 & Inspección visual del estado, conexionado, porcelana, contactor, etc. & $M$ \\
\hline
\end{tabular}




\begin{tabular}{|l|l|l|}
\hline $\mathbf{2}$ & Chequear las conexiones de líneas y puesta a tierra. & $\boldsymbol{M}$ \\
\hline $\mathbf{3}$ & Chequear estado de porcelana y limpieza. & $\boldsymbol{A}$ \\
\hline $\mathbf{4}$ & Verificar el ajuste de anillos equipotenciales, conexiones aéreas y puesta a tierra. & $\boldsymbol{A}$ \\
\hline $\mathbf{5}$ & Medición de la resistencia de aislamiento. & $\boldsymbol{A}$ \\
\hline $\mathbf{6}$ & Pruebas de factor de potencia. & $\mathbf{2} \boldsymbol{A}$ \\
\hline
\end{tabular}

Fuente: Instituto Ecuatoriano de Electrificación (INECEL)

Tabla 18 Banco de tareas para Mantenimiento Preventivo a Barrajes, estructuras y puesta a tierra.

\begin{tabular}{|l|l|l|}
\hline \multicolumn{2}{|l|}{ Banco de tareas para Barrajes, estructuras y puesta a tierra. } & Frecuencia \\
\hline $\mathbf{1}$ & Chequeo de aisladores, barras, conexionado aéreo, cable de guarda, estructuras. & $\boldsymbol{A}$ \\
\hline $\mathbf{2}$ & Medir resistencia de aislamiento. & $\boldsymbol{A}$ \\
\hline $\mathbf{3}$ & Chequeo de las conexiones de puesta a tierra, estructuras, cerramientos, puertas. & $\boldsymbol{A}$ \\
\hline $\mathbf{4}$ & Verificar por muestreo la conexión de puesta a tierra a la maya de tierra. & $\mathbf{2 A}$ \\
\hline $\mathbf{5}$ & Realizar pruebas de medición de resistencia de tierra a la maya de tierra. & $\mathbf{2 A}$ \\
\hline $\mathbf{6}$ & Realizar pruebas de termovisión. & $\mathbf{2 A}$ \\
\hline
\end{tabular}

Fuente: Instituto Ecuatoriano de Electrificación (INECEL)

Tabla 19 Banco de tareas para Mantenimiento Preventivo de Bancos de Capacitores.

\begin{tabular}{|l|l|l|}
\hline \multicolumn{2}{|l|}{ Banco de tareas para Banco de Capacitores. } & Frecuencia \\
\hline $\mathbf{1}$ & $\begin{array}{l}\text { Realizar una inspección visual de los bushings, tanque, niveles de aceite, estructuras y } \\
\text { puesta a tierra. }\end{array}$ & $\boldsymbol{M}$ \\
\hline $\mathbf{2}$ & Chequear la porcelana y observas despostillados, rajaduras, cementación y limpieza. & $\boldsymbol{A}$ \\
\hline $\mathbf{3}$ & Chequear tuberías, accesorios, conexionado y fusibles. & $\boldsymbol{A}$ \\
\hline $\mathbf{4}$ & Pruebas del factor de potencia. & $\mathbf{2} \boldsymbol{A}$ \\
\hline $\mathbf{5}$ & Pruebas de resistencia de aislamiento. & $\boldsymbol{A}$ \\
\hline $\mathbf{6}$ & Medir la corriente del neutro. & $\boldsymbol{A}$ \\
\hline $\mathbf{7}$ & Limpieza de porcelanas, tanques, fusibles, chequeo de ajuste. & $\mathbf{3} \boldsymbol{A}$ \\
\hline $\mathbf{8}$ & Coger fallas de pintura. & $\boldsymbol{A}$ \\
\hline
\end{tabular}

Fuente: Instituto Ecuatoriano de Electrificación (INECEL) 
Tabla 20 Banco de tareas para Mantenimiento Preventivo a Transformadores para Servicios Auxiliares.

\begin{tabular}{|l|l|l|}
\hline \multicolumn{2}{|l|}{ Banco de tareas a Transformadores para Servicios Auxiliares. } & Frecuencia \\
\hline $\mathbf{1}$ & Verificar el estado de bushings y accesorios. & M \\
\hline 2 & Inspección del transformador, limpieza y ajuste del conexionado. & A \\
\hline 3 & Pruebas dieléctricas del aceite. & A \\
\hline 4 & Medición de resistencia de aislamiento. & A \\
\hline $\mathbf{5}$ & Coger fallas de pintura. & A \\
\hline
\end{tabular}

Fuente: Instituto Ecuatoriano de Electrificación (INECEL)

Tabla 21 Banco de tareas para Mantenimiento Preventivo en Cables y Ductos.

\begin{tabular}{|l|l|l|}
\hline \multicolumn{2}{|l|}{ Banco de tareas para Cables y Ductos. } & Frecuencia \\
\hline $\mathbf{1}$ & $\begin{array}{l}\text { Chequear la condición de los cables en canaletas, ductos, pozos de revisión (roturas, } \\
\text { corrosión, señales de calentamiento, etc.). }\end{array}$ & $\boldsymbol{A}$ \\
\hline $\mathbf{2}$ & Inspeccionar la cubierta de los cables en las curvaturas de las canaletas. & $\boldsymbol{A}$ \\
\hline $\mathbf{3}$ & $\begin{array}{l}\text { Chequear que las canaletas, ductos y pozos de revisión estén sin basura, polvo, } \\
\text { humedad, verificar que los drenajes estén limpios. }\end{array}$ & $\boldsymbol{A}$ \\
\hline
\end{tabular}

Fuente: Instituto Ecuatoriano de Electrificación (INECEL)

Tabla 22 Banco de tareas para Mantenimiento Preventivo a Tableros Dúplex (Protección, control y medición).

\begin{tabular}{|l|l|l|}
\hline \multicolumn{2}{|c|}{ Banco de tareas para Tableros Dúplex (Protección, control y medición). } & Frecuencia \\
\hline $\mathbf{1}$ & $\begin{array}{l}\text { Inspección visual del estado de instrumentos de medición, protección, de control, } \\
\text { señalización (ruidos anormales, vibraciones, etc.). }\end{array}$ & $\boldsymbol{M}$ \\
\hline $\mathbf{2}$ & Inspección del conexionado y ajuste. & $\boldsymbol{A}$ \\
\hline $\mathbf{3}$ & Verificación de estado de cuadros de alarmas y bocinas. & $\boldsymbol{M}$ \\
\hline $\mathbf{4}$ & Calibración de instrumentos de medición. & $\mathbf{2} \boldsymbol{A}$ \\
\hline $\mathbf{5}$ & Verificar el estado de fusibles, contactores, térmicos, manillas de operación, selectores. & $\boldsymbol{A}$ \\
\hline $\mathbf{6}$ & Mantenimiento preventivo de relés. & $\boldsymbol{A}$ \\
\hline $\mathbf{7}$ & Limpieza general de tableros. & $\boldsymbol{M}$ \\
\hline
\end{tabular}

Fuente: Instituto Ecuatoriano de Electrificación (INECEL) 
Tabla 23 Banco de tareas para Mantenimiento Preventivo a Tableros de Servicios Auxiliares (C.A. y C.C.) Cargadores de Baterías.

\begin{tabular}{|l|l|l|}
\hline \multicolumn{2}{|l|}{$\begin{array}{l}\text { Banco de tareas para Tableros de Servicios Auxiliares (C.A. y C.C.) Cargadores de } \\
\text { Baterias. }\end{array}$} & Frecuencia \\
\hline $\mathbf{1}$ & Verificación visual de instrumentos de medición, protección, control, señalización. & $\boldsymbol{M}$ \\
\hline $\mathbf{2}$ & Verificación del estado del conexionado, limpieza y ajuste. & $\boldsymbol{A}$ \\
\hline $\mathbf{3}$ & Inspección general del cargador de baterías (medición, control y protección). & $\boldsymbol{M}$ \\
\hline $\mathbf{4}$ & Verificación del estado de cuadros de alarmas y bocinas. & $\boldsymbol{M}$ \\
\hline $\mathbf{5}$ & Verificar el estado de fusibles, contactores, térmicos, manillas de operación, selectores. & $\boldsymbol{A}$ \\
\hline $\mathbf{6}$ & Calibración de instrumentos de medición. & $\mathbf{A}$ \\
\hline $\mathbf{7}$ & Limpieza general de tableros. & $\boldsymbol{M}$ \\
\hline $\mathbf{8}$ & Comprobar y calibrar voltajes de ecualización y flotación en cargadores. & \\
\hline
\end{tabular}

Fuente: Instituto Ecuatoriano de Electrificación (INECEL)

Tabla 24 Banco de Tareas para Mantenimiento Preventivo de las Baterías

\begin{tabular}{|c|c|c|}
\hline \multicolumn{2}{|c|}{ Banco de Tareas para Baterías. } & \multirow{2}{*}{$\begin{array}{l}\text { Frecuencia } \\
M\end{array}$} \\
\hline 1 & Verificar que el voltaje D.C. este dentro del rango normal. & \\
\hline 2 & $\begin{array}{l}\text { Verificar que la lectura del amperímetro del cargador este dentro de los límites } \\
\text { normales. }\end{array}$ & $M$ \\
\hline 3 & Registrar la densidad, voltaje y temperatura de la celda piloto. & $M$ \\
\hline 4 & Control de temperatura y densidad del banco (cada celda). & $S M$ \\
\hline 5 & Verificar los niveles de electrolito y añadir agua destilada si es necesario. & $M$ \\
\hline 6 & Aplicar una carga igualadora por el tiempo que recomiende el fabricante. & $\boldsymbol{A}$ \\
\hline 7 & Verificar el estado de las placas de las baterías. & $M$ \\
\hline 8 & Efectuar la limpieza de las cubetas. & $\boldsymbol{A}$ \\
\hline 9 & Limpieza de conexiones y cubrirlas con vaselina u otro lubricante apropiado. & $\boldsymbol{A}$ \\
\hline 10 & Inspeccionar y reparar rajaduras de las cubetas. & $\boldsymbol{C R}$ \\
\hline 11 & Verificar la precisión de los instrumentos. & $\boldsymbol{A}$ \\
\hline 12 & $\begin{array}{l}\text { Con el cargador desconectado, operar equipos para prueba con servicio de emergencia } \\
\text { de baterías. }\end{array}$ & $\boldsymbol{A}$ \\
\hline 13 & Verificar la operación de las alarmas (bajo voltaje). & $\boldsymbol{A}$ \\
\hline
\end{tabular}

Fuente: Instituto Ecuatoriano de Electrificación (INECEL) 
Tabla 25 Banco de tareas para Mantenimiento Predictivo en el Sistema de Aire Acondicionado.

\begin{tabular}{|c|c|c|}
\hline \multicolumn{2}{|r|}{ Banco de tareas para Sistema de Aire Acondicionado. } & Frecuencia \\
\hline 1 & $\begin{array}{l}\text { Chequear los motores para detectar polvo, agua, limallas, limpieza y buen } \\
\text { funcionamiento. }\end{array}$ & $M$ \\
\hline 2 & Chequear lubricación en rodamientos, preferible con el motor parado. & $\boldsymbol{A}$ \\
\hline 3 & Medición de la resistencia de aislamiento de los bobinados. & $\boldsymbol{A}$ \\
\hline 4 & $\begin{array}{l}\text { Con el motor en operación observar su comportamiento, aceleración, ruidos inusuales, } \\
\text { vibración, chisporroteo, humedad. }\end{array}$ & $M$ \\
\hline 5 & Inspeccionar acoplamiento, cadenas, observar alineación, vibración, ruido. & $M$ \\
\hline 6 & Chequear calefactores. & $M$ \\
\hline
\end{tabular}

Fuente: Instituto Ecuatoriano de Electrificación (INECEL)

Tabla 26 Banco de tareas para Mantenimiento Correctivo en Transformadores de Potencia.

\begin{tabular}{|l|l|l|}
\hline \multicolumn{2}{|l|}{ Banco de tareas para Transformadores de Potencia. } & Frecuencia \\
\hline $\mathbf{1}$ & Cambio de radiadores averiados. & $\boldsymbol{C R}$ \\
\hline $\mathbf{2}$ & Cambio de bujes averiados. & $\boldsymbol{C R}$ \\
\hline $\mathbf{3}$ & Cambio de ventiladores. & $\boldsymbol{C R}$ \\
\hline $\mathbf{4}$ & Cambio del regulador. & $\boldsymbol{C R}$ \\
\hline $\mathbf{5}$ & Cambio del aceite. & $\boldsymbol{C R}$ \\
\hline $\mathbf{6}$ & Cambio del Transformador de potencia. & $\boldsymbol{C R}$ \\
\hline
\end{tabular}

Fuente: http://dispac.com.co/wp-content/uploads/2015/05/ANEXO-18-A-MANUAL-DEMANTENIMIENTO-PARA-SUBESTACIONES.pdf

Tabla 27 Banco de tareas para Mantenimiento Correctivo de Interruptores de Potencia.

\begin{tabular}{|l|l|c|}
\hline \multicolumn{2}{|c|}{ Banco de tareas para Interruptores de Potencia. } & Frecuencia \\
\hline $\mathbf{1}$ & Cambio de contactos de potencia Fijo y Móvil. & CR \\
\hline 2 & Cambio del SF6. & $\boldsymbol{C R}$ \\
\hline 3 & Cambio del mecanismo de operación. & $\boldsymbol{C R}$ \\
\hline 4 & Cambio del Interruptor de Potencia. & $\boldsymbol{C R}$ \\
\hline
\end{tabular}

Fuente: http://dispac.com.co/wp-content/uploads/2015/05/ANEXO-18-A-MANUAL-DEMANTENIMIENTO-PARA-SUBESTACIONES.pdf 
Tabla 28 Banco de tareas para Mantenimiento Correctivo de Seccionadores de Potencia.

\begin{tabular}{|l|l|l|}
\hline \multicolumn{2}{|l|}{ Banco de tareas para Seccionadores de Potencia. } & Frecuencia \\
\hline $\mathbf{1}$ & Cambio de contactos. & $\boldsymbol{C R}$ \\
\hline $\mathbf{2}$ & Cambio del mecanismo de operación. & $\boldsymbol{C R}$ \\
\hline $\mathbf{3}$ & Cambio de Brazos de corriente. & $\boldsymbol{C R}$ \\
\hline $\mathbf{4}$ & Cambio de aisladores soporte. & $\boldsymbol{C R}$ \\
\hline $\mathbf{5}$ & Cambio de sistema de engranaje. & $\mathbf{C R}$ \\
\hline $\mathbf{6}$ & Cambio del Seccionador de Potencia. & $\mathbf{C R}$ \\
\hline
\end{tabular}

Fuente: http://dispac.com.co/wp-content/uploads/2015/05/ANEXO-18-A-MANUAL-DEMANTENIMIENTO-PARA-SUBESTACIONES.pdf

Tabla 29 Banco de tareas para Mantenimiento Correctivo a Transformadores de Corriente.

\begin{tabular}{|l|l|l|}
\hline \multicolumn{2}{|l|}{ Banco de tareas para Transformadores de Corriente. } & Frecuencia \\
\hline 1 & Cambio del Aceite. & CR \\
\hline 2 & Cambio del Transformador de Corriente. & CR \\
\hline
\end{tabular}

Fuente: http://dispac.com.co/wp-content/uploads/2015/05/ANEXO-18-A-MANUAL-DEMANTENIMIENTO-PARA-SUBESTACIONES.pdf

Tabla 30 Banco de tareas para Mantenimiento Correctivo a Transformadores de Potencial o de Tensión.

\begin{tabular}{|l|l|l|}
\hline \multicolumn{2}{|l|}{ Banco de tareas para Transformadores de Potencial o de Tensión. } & Frecuencia \\
\hline 1 & Cambio del Aceite. & CR \\
\hline 2 & Cambio del Transformador de Potencial o Tensión. & CR \\
\hline
\end{tabular}

Fuente: http://dispac.com.co/wp-content/uploads/2015/05/ANEXO-18-A-MANUAL-DEMANTENIMIENTO-PARA-SUBESTACIONES.pdf

Tabla 31 Banco de tareas para Mantenimiento Correctivo a los Pararrayos.

\begin{tabular}{|l|l|l|}
\hline \multicolumn{2}{|l|}{ Banco de tareas para los Pararrayos. } & Frecuencia \\
\hline 1 & Cambio de Pararrayo. & CR \\
\hline
\end{tabular}

Fuente: http://dispac.com.co/wp-content/uploads/2015/05/ANEXO-18-A-MANUAL-DEMANTENIMIENTO-PARA-SUBESTACIONES.pdf 
Tabla 32 Banco de tareas para Mantenimiento Correctivo a la Malla de Puesta a Tierra.

\begin{tabular}{|l|l|l|}
\hline \multicolumn{2}{|l|}{ Banco de tareas para Malla de Puesta a Tierra. } & Frecuencia \\
\hline $\mathbf{1}$ & Cambio o refuerzo del conductor de la malla y de las colas. & $\mathbf{C R}$ \\
\hline $\mathbf{2}$ & Cambio de las conexiones. & $\mathbf{C R}$ \\
\hline $\mathbf{3}$ & Reposición de gravilla. & $\mathbf{C R}$ \\
\hline
\end{tabular}

Fuente: http://dispac.com.co/wp-content/uploads/2015/05/ANEXO-18-A-MANUAL-DEMANTENIMIENTO-PARA-SUBESTACIONES.pdf

Tabla 33 Banco de tareas para Mantenimiento Correctivo a Barrajes y conectores.

\begin{tabular}{|l|l|l|}
\hline \multicolumn{2}{|l|}{ Banco de tareas para Barrajes y conectores. } & Frecuencia \\
\hline 1 & Cambio del conductor o del barraje tubular. & CR \\
\hline
\end{tabular}

Fuente: http://dispac.com.co/wp-content/uploads/2015/05/ANEXO-18-A-MANUAL-DEMANTENIMIENTO-PARA-SUBESTACIONES.pdf

Tabla 34 Banco de tareas para Mantenimiento Correctivo a Tableros de control, medidas y protecciones.

\begin{tabular}{|l|l|l|}
\hline \multicolumn{2}{|l|}{ Banco de tareas para Tableros de control, medidas y protecciones. } & Frecuencia \\
\hline $\mathbf{1}$ & Cambio de borneras. & CR \\
\hline 2 & Cambio del tablero. & CR \\
\hline
\end{tabular}

Fuente: http://dispac.com.co/wp-content/uploads/2015/05/ANEXO-18-A-MANUAL-DEMANTENIMIENTO-PARA-SUBESTACIONES.pdf

Tabla 35 Banco de tareas para Mantenimiento Correctivo en Celdas de alto voltaje.

\begin{tabular}{|l|l|l|}
\hline \multicolumn{2}{|l|}{ Banco de tareas para Celdas de alto voltaje. } & Frecuencia \\
\hline $\mathbf{1}$ & Cambio de Interruptor de potencia. & $\boldsymbol{C R}$ \\
\hline $\mathbf{2}$ & Cambio de borneras. & $\boldsymbol{C R}$ \\
\hline 3 & Cambio de aisladores soporte. & $\boldsymbol{C R}$ \\
\hline 4 & Cambio de la Celda. & $\mathbf{C R}$ \\
\hline
\end{tabular}

Fuente: http://dispac.com.co/wp-content/uploads/2015/05/ANEXO-18-A-MANUAL-DEMANTENIMIENTO-PARA-SUBESTACIONES.pdf 
Tabla 36 Banco de tareas para Mantenimiento Correctivo a los Bancos de Baterías.

\begin{tabular}{|l|l|c|}
\hline \multicolumn{2}{|l|}{ Banco de tareas para los Bancos de Baterías. } & Frecuencia \\
\hline 1 & Cambio de banco de baterías. & CR \\
\hline
\end{tabular}

Fuente: http://dispac.com.co/wp-content/uploads/2015/05/ANEXO-18-A-MANUAL-DE-

MANTENIMIENTO-PARA-SUBESTACIONES.pdf

Tabla 37 Banco de tareas para Mantenimiento Predictivo en las Estructuras.

\begin{tabular}{|c|c|c|}
\hline \multicolumn{2}{|c|}{ Banco de tareas para las Estructuras. } & Frecuencia \\
\hline 1 & $\begin{array}{l}\text { Inspección visual sobre la condición de las fundaciones y terreno alrededor de las } \\
\text { torres, tales como suelos dispersivos, falla de talud ante eventos sísmicos y erosión del } \\
\text { terreno. }\end{array}$ & $\boldsymbol{A}$ \\
\hline 2 & $\begin{array}{l}\text { Inspección visual para determinar en las estructuras la existencia de elementos y } \\
\text { tornillos dañados, perdida del galvanizado, manchas de oxidación, surgimiento de } \\
\text { burbujas y escamas, expansión y pérdida de su sección. }\end{array}$ & $A$ \\
\hline
\end{tabular}

Fuente: http://repositorio.utp.edu.co/dspace/bitstream/handle/11059/1064/6213192G984.pdf?sequence=1

Tabla 38 Banco de tareas para Mantenimiento Predictivo en los Aislamientos.

\begin{tabular}{|c|c|c|}
\hline \multicolumn{2}{|c|}{ Banco de tareas para los aislamientos. } & \multirow{2}{*}{ Frecuencia } \\
\hline 1 & Inspección termográfica. & \\
\hline 2 & Localización de descargas parciales. & $A$ \\
\hline 3 & $\begin{array}{l}\text { Inspección visual para detectar la existencia sobre el material aislante lineas de fugas } \\
\text { estrechas de forma continua o discontinua y líneas de fugas anchas y continuas. }\end{array}$ & $A$ \\
\hline 4 & $\begin{array}{l}\text { Inspección visual para detectar la existencia sobre el material aislante líneas de fugas } \\
\text { anchas y continuas. }\end{array}$ & $\boldsymbol{A}$ \\
\hline 5 & $\begin{array}{l}\text { Inspección visual para detectar en el caso de aisladores de porcelana, la existencia de } \\
\text { rotura o agrietamiento en la superficie aislante. }\end{array}$ & $A$ \\
\hline 6 & $\begin{array}{l}\text { Inspección visual para detectar en el caso de aisladores de vidrio la carencia de la parte } \\
\text { aislante. }\end{array}$ & $A$ \\
\hline 7 & $\begin{array}{l}\text { Inspección visual del elemento metálico para determinar la existencia de pérdida del } \\
\text { galvanizado, manchas de oxidación, surgimiento de burbujas y escamas, expansión y } \\
\text { pérdida de su sección. }\end{array}$ & $A$ \\
\hline
\end{tabular}

Fuente: http://repositorio.utp.edu.co/dspace/bitstream/handle/11059/1064/6213192G984.pdf?sequence=1

Tabla 39 Banco de tareas para Mantenimiento Predictivo a los cables conductores. 


\begin{tabular}{|l|l|l|}
\hline $\mathbf{1}$ & Inspección termográfica. & $\boldsymbol{A}$ \\
\hline $\mathbf{2}$ & $\begin{array}{l}\text { Inspección visual para detectar la existencia en el conductor líneas de fugas estrechas } \\
\text { de forma continua o discontinua y lineas de fugas anchas y continuas. }\end{array}$ & $\boldsymbol{A}$ \\
\hline $\mathbf{3}$ & $\begin{array}{l}\text { Inspección visual para detectar la existencia en el conductor líneas de fugas anchas y } \\
\text { continuas. }\end{array}$ & $\boldsymbol{A}$ \\
\hline
\end{tabular}

Fuente: http://repositorio.utp.edu.co/dspace/bitstream/handle/11059/1064/6213192G984.pdf?sequence=1

Tabla 40 Banco de tareas para Mantenimiento Predictivo de los Sistemas de Protección.

\begin{tabular}{|l|l|l|}
\hline \multicolumn{2}{|l|}{ Banco de tareas para los Sistemas de Protección. } & Frecuencia \\
\hline $\mathbf{1}$ & Inspección termográfica. & $\boldsymbol{A}$ \\
\hline $\mathbf{2}$ & Localización de descargas parciales. & $\boldsymbol{A}$ \\
\hline $\mathbf{3}$ & Medida de la resistencia de puesta a tierra. & $\boldsymbol{A}$ \\
\hline $\mathbf{4}$ & Inspección visual de todas las partes componentes del cable guarda. & $\boldsymbol{A}$ \\
\hline
\end{tabular}

Fuente: http://repositorio.utp.edu.co/dspace/bitstream/handle/11059/1064/6213192G984.pdf?sequence=1

Tabla 41 Banco de tareas para Mantenimiento Preventivo de Fajas de Servidumbre.

\begin{tabular}{|l|l|l|}
\hline \multicolumn{2}{|l|}{ Banco de tareas en fajas de servidumbre. } & Frecuencia \\
\hline 1 & Realizar rocería, poda o tala de árboles. & SM \\
\hline 2 & Limpieza de las bases o sitios de torres. & SM \\
\hline 3 & Verificar que no exista deforestación indiscriminada. & SM \\
\hline
\end{tabular}

Fuente:http://www.siame.gov.co/siame/documentos/Guias_Ambientales/Gu\%C3\%ADas\%20Resoluci\%C 3\%B3n\%201023\%20del\%2028\%20de\%20julio\%20de\%202005/SECTOR\%20ENERG\%C3\%89TICO/Gu ia\%20para\%20proyectos\%20de \%20transmisi\%C3\%B3n\%20el\%C3\%A9ctrica.pdf

Tabla 42 Banco de tareas para Mantenimiento Preventivo en las Estructuras.

\begin{tabular}{|c|c|c|}
\hline \multicolumn{2}{|c|}{ Banco de tareas para las Estructuras. } & Frecuencia \\
\hline 1 & Cambio o refuerzo de estructuras, o de algunos de sus elementos. & $C R$ \\
\hline 2 & $\begin{array}{l}\text { Tratamiento de protección anticorrosiva, especialmente en las bases y señalización de } \\
\text { estructuras. }\end{array}$ & CR \\
\hline 3 & $\begin{array}{l}\text { Realizar obras de protección como trinchos, muros de contención, gaviones, cunetas, } \\
\text { empradizados entre otras en caso de anomalías tales como erosiones, riesgos de } \\
\text { avalancha o derrumbe, que atenten contra la estabilidad de los sitios de la torre o de las } \\
\text { zonas circundantes. }\end{array}$ & $C R$ \\
\hline
\end{tabular}


Fuente:http:/www.siame.gov.co/siame/documentos/Guias_Ambientales/Gu\%C3\%ADas\%20Resoluci\%C 3\%B3n\%201023\%20del\%2028\%20de\%20julio\%20de\%202005/SECTOR\%20ENERG\%C3\%89TICO/Gu ia\%20para\%20proyectos\%20de \%20transmisi\%C3\%B3n\%20el\%C3\%A9ctrica.pdf

Tabla 43 Banco de tareas para Mantenimiento Preventivo en los Aislamientos.

\begin{tabular}{|l|l|l|}
\hline \multicolumn{2}{|l|}{ Banco de tareas para los aislamientos. } & Frecuencia \\
\hline $\mathbf{1}$ & Cambio de aisladores rotos. & CR \\
\hline 2 & Cambio de accesorios defectuosos de las cadenas de aisladores. & CR \\
\hline 3 & Lavado de los aisladores. & $\boldsymbol{A}$ \\
\hline
\end{tabular}

Fuente:http:/www.siame.gov.co/siame/documentos/Guias_Ambientales/Gu\%C3\%ADas\%20Resoluci\%C 3\%B3n\%201023\%20del\%2028\%20de\%20julio\%20de\%202005/SECTOR\%20ENERG\%C3\%89TICO/Gu ia\%20para\%20proyectos\%20de \%20transmisi\%C3\%B3n\%20el\%C3\%A9ctrica.pdf

Tabla 44 Banco de tareas para Mantenimiento Preventivo en los Cables Conductores.

\begin{tabular}{|l|l|l|}
\hline \multicolumn{2}{|l|}{ Banco de tareas para los Cables Conductores. } & Frecuencia \\
\hline $\mathbf{1}$ & Cambios de empalmes, blindajes o camisas de reparación instalados. & CR \\
\hline 2 & Cambio de uno o varios conductores defectuosos. & CR \\
\hline 3 & $\begin{array}{l}\text { Instalación de balizas de señalización. } \\
\text { Verificar conexiones flojas, rotas, fisuradas, desgastadas, deformadas o corroidas, } \\
\text { pines faltantes que puedan generar desprendimiento del herraje con el conductor. }\end{array}$ & $\boldsymbol{A}$ \\
\hline
\end{tabular}

Fuente:http://www.siame.gov.co/siame/documentos/Guias_Ambientales/Gu\%C3\%ADas\%20Resoluci\%C 3\%B3n\%201023\%20del\%2028\%20de\%20julio\%20de\%202005/SECTOR\%20ENERG\%C3\%89TICO/Gu ia\%20para\%20proyectos\%20de \%20transmisi\%C3\%B3n\%20el\%C3\%A9ctrica.pdf

Tabla 45 Banco de tareas para Mantenimiento Preventivo en los Sistemas de Protección.

\begin{tabular}{|l|l|l|}
\hline \multicolumn{2}{|l|}{ Banco de tareas para los Sistemas de Protección. } & Frecuencia \\
\hline $\mathbf{1}$ & Cambio de accesorios defectuosos de cable de guarda. & CR \\
\hline 2 & Cambio de accesorios defectuosos de puestas a tierra. & CR \\
\hline 3 & $\begin{array}{l}\text { Verificar el ajuste entre la estructura, los cables de puesta a tierra y los elementos de } \\
\text { conexión a tierra. }\end{array}$ & $\boldsymbol{A}$ \\
\hline 4 & Verificar las distancias entre los conductores y cables de guarda. & $\boldsymbol{A}$ \\
\hline
\end{tabular}


Fuente:http:/www.siame.gov.co/siame/documentos/Guias_Ambientales/Gu\%C3\%ADas\%20Resoluci\%C 3\%B3n\%201023\%20del\%2028\%20de\%20julio\%20de\%202005/SECTOR\%20ENERG\%C3\%89TICO/Gu ia\%20para\%20proyectos\%20de \%20transmisi\%C3\%B3n\%20el\%C3\%A9ctrica.pdf

Tabla 46 Banco de tareas para Mantenimiento Correctivo en las Fajas de Servidumbre.

\begin{tabular}{|l|l|c|}
\hline \multicolumn{2}{|l|}{ Banco de tareas para la faja de servidumbre. } & Frecuencia \\
\hline 1 & Ejecución de rocería, poda o tala de árboles. & CR \\
\hline
\end{tabular}

Fuente:http://www.siame.gov.co/siame/documentos/Guias_Ambientales/Gu\%C3\%ADas\%20Resoluci\%C 3\%B3n\%201023\%20del\%2028\%20de\%20julio\%20de\%202005/SECTOR\%20ENERG\%C3\%89TICO/Gu ia\%20para\%20proyectos\%20de \%20transmisi\%C3\%B3n\%20el\%C3\%A9ctrica.pdf

Tabla 47 Banco de tareas para Mantenimiento Correctivo en las Estructuras.

\begin{tabular}{|l|l|l|}
\hline \multicolumn{2}{|l|}{ Banco de tareas para las Estructuras. } & Frecuencia \\
\hline $\boldsymbol{1}$ & Cambio total o parcial de estructura defectuosa. & $\boldsymbol{C R}$ \\
\hline 2 & Corregir fallas de pintura de cualquier tipo. & $\boldsymbol{C R}$ \\
\hline
\end{tabular}

Fuente:http://www.siame.gov.co/siame/documentos/Guias_Ambientales/Gu\%C3\%ADas\%20Resoluci\%C 3\%B3n\%201023\%20del\%2028\%20de\%20julio\%20de\%202005/SECTOR\%20ENERG\%C3\%89TICO/Gu ia\%20para\%20proyectos\%20de\%20transmisi\%C3\%B3n\%20el\%C3\%A9ctrica.pdf

Tabla 48 Banco de tareas para Mantenimiento Correctivo en los Aisladores.

\begin{tabular}{|c|l|c|}
\hline \multicolumn{2}{|l|}{ Banco de tareas para los aislamientos. } & Frecuencia \\
\hline $\mathbf{1}$ & Cambio de cadenas de aisladores defectuosos. & CR \\
\hline 2 & $\begin{array}{l}\text { En aisladores de Polimero, realizar cambio del mismo al existir ausencia mayor al 10\% } \\
\text { de sus aletas. }\end{array}$ & $\boldsymbol{C R}$ \\
\hline $\mathbf{3}$ & Cambio de herrajes de suspensión y retención & $\boldsymbol{C R}$ \\
\hline
\end{tabular}

Fuente:http:/www.siame.gov.co/siame/documentos/Guias_Ambientales/Gu\%C3\%ADas\%20Resoluci\%C 3\%B3n\%201023\%20del\%2028\%20de\%20julio\%20de\%202005/SECTOR\%20ENERG\%C3\%89TICO/Gu ia\%20para\%20proyectos\%20de\%20transmisi\%C3\%B3n\%20el\%C3\%A9ctrica.pdf

Tabla 49 Banco de tareas para Mantenimiento Correctivo en los Cables Conductores.

\begin{tabular}{|l|l|c|}
\hline \multicolumn{2}{|l|}{ Banco de tareas para los Cables Conductores. } & Frecuencia \\
\hline 1 & Reparación de conductor con empalme, blindaje y manguito & CR \\
\hline 2 & Cambio de conductor con todos sus accesorios. & CR \\
\hline
\end{tabular}


Fuente:http://www.siame.gov.co/siame/documentos/Guias_Ambientales/Gu\%C3\%ADas\%20Resoluci\%C 3\%B3n\%201023\%20del\%2028\%20de\%20julio\%20de\%202005/SECTOR\%20ENERG\%C3\%89TICO/Gu ia\%20para $\% 20$ proyectos\%20de $\% 20$ transmisi $\%$ C3\%B3n\%20el\%C3\%A9ctrica.pdf

Tabla 50 Banco de tareas para Mantenimiento Correctivo en los Sistemas de Protección.

\begin{tabular}{|l|l|l|}
\hline \multicolumn{2}{|l|}{ Banco de tareas para los Sistemas de Protección. } & Frecuencia \\
\hline $\mathbf{1}$ & Reparación de cable de guarda con empalme y blindaje & CR \\
\hline 2 & Mejoramiento del sistema de puesta a tierra. & $\boldsymbol{C R}$ \\
\hline 3 & Sustitución del cable de guarda. & $\boldsymbol{C R}$ \\
\hline
\end{tabular}

Fuente:http:/www.siame.gov.co/siame/documentos/Guias_Ambientales/Gu\%C3\%ADas\%20Resoluci\%C 3\%B3n\%201023\%20del\%2028\%20de\%20julio\%20de\%202005/SECTOR\%20ENERG\%C3\%89TICO/Gu ia\%20para\%20proyectos\%20de \%20transmisi\%C3\%B3n\%20el\%C3\%A9ctrica.pdf

Y en tercer lugar se elabora la Programación de Mantenimiento Predictivo y Preventivo que será útil para el Sistema de Subtransmisión de CNEL EP Unidad de Negocio Esmeraldas.

\section{Conclusiones}

Se realizó un modelo de gestión de mantenimiento idóneo para las subestaciones y líneas de subtransmisión de CNEL EP Unidad de Negocio Esmeraldas, enmarcado en un cronograma de actividades listas para su ejecución.

En las visitas al Sistema de Subtransmisión de CNEL EP Unidad de Negocio Esmeraldas se observó que no todos los sistemas se encuentran en condiciones óptimas, lo que refleja la ausencia de un adecuado plan de mantenimiento en ellas, ya que no cumplen con todas las actividades que se deben realizar a dicho sistema.

Se pudo inferir en la importancia y la cantidad de tareas o actividades que se deben de realizar para cada equipo del sistema de subtransmisión, por ende, la Programación de Mantenimiento realizada en el presente proyecto será muy útil a CNEL EP Unidad de Negocio Esmeraldas. 
Se propone un cronograma de actividades que contemplan los mantenimientos adecuados para cada equipo del sistema de subtransmisión, el mismo que puede ser adaptado o modificado en base a los recursos y herramientas con los que cuenta CNEL EP Unidad de Negocio Esmeraldas.

\section{Referencias Bibliográficas}

FRANCISCO, C. J., \& OCHOA, J. A. (2008). OCHCAR. Recuperado el 02 de febrero de 2016, de

HTTP:/MRIUC.BC.UC.EDU.VE/BITSTREAM/HANDLE/123456789/53/OCHCAR.PDF?SEQ $\mathrm{UENCE}=4$

MARTINEZ, F. M. (2010). BENEFICIOS DE REDUCIR MANTENIMIENTO PREVENTIVO. $\begin{array}{lllllll}\text { Recuperado el de } & 02 \text { febrero de }\end{array}$ HTTP://REPOSITORIO.UIS.EDU.CO/JSPUI/BITSTREAM/123456789/7722/2/134729.PDF

PEREZ, J. G. (2011). DESCRIPCION Y FUNCION DEL EQUIPO DE UNA SUBESTACION ELECTRICA. Recuperado el 02 de febrero de 2016, de HTTP:/CDIGITAL.UV.MX/BITSTREAM/123456789/29582/1/MARPEREZYVIDALLOPEZ. PDF

SANCLEMENTE, J. P., \& NIETO ALVARADO, L. G. (2010). UPS-GT000156. Recuperado el 02 de febrero $\quad$ de $2016, \quad$ de HTTP://DSPACE.UPS.EDU.EC/BITSTREAM/123456789/2119/15/UPS-GT000156.PDF

SENPLADES. (24 de Junio de 2013). Plan Buen Vivir 2013-2017. Obtenido de Políticas y lineamientos estratégicos 11.1: http://www.planificacion.gob.ec/

www.solomantenimiento,com. (s.f). DICCIONARIO TECNICO MECANICO - DICCIONARIO DE MECANICA INDUSTRIAL. Obtenido de HTTP://WWW.SOLOMANTENIMIENTO.COM/DICCIONARIO_MECANICO.HTM 